\title{
Inovação em Projetos de Software aplicada a Soluções Educacionais
}

\author{
Ricardo André Cavalcante de Souza \\ Departamento de Estatística e Informática (DEINFO) - Universidade Federal Rural de \\ Pernambuco (UFRPE) - Recife/PE - Brasil \\ ricardo. souzadufrpe.br
}

\begin{abstract}
This work presents the experience of the application of a teachinglearning approach aiming to provide knowledge and to foster abilities for the students necessary for: deep understanding of a problem; formulation of a relevant challenge; identification of innovation opportunities; and design and prototyping a solution through creativity and teamwork. This approach was experimented in the context of developing software-based solutions to solve problems related to education.
\end{abstract}

Resumo. Este trabalho apresenta o relato de experiência da aplicação de uma abordagem de ensino-aprendizagem visando prover conhecimentos e fomentar habilidades aos estudantes necessários para: entendimento aprofundado de um problema; formulação de um desafio relevante; identificação de oportunidades de inovação; e design e prototipação de uma solução por meio da criatividade e trabalho em equipe. Esta abordagem foi experimentada no contexto de desenvolvimento de soluções baseadas em software para resolver problemas relacionados à educação.

\section{Introdução}

As abordagens de ensino tradicionais são caracterizadas como "transmissão direta", isto é, os professores comunicam conhecimento de um modo estruturado, demonstram e explicam soluções. As abordagens de ensino-aprendizagem inovadoras, por outro lado, são caracterizadas como centradas no estudante e a ênfase está no desenvolvimento de habilidades para "aprender-a-aprender" (OECD, 2014). Nestas, os professores assumem papéis diferentes, movendo-se do ensino "na frente da sala de aula" para participação ativa no processo de aprendizagem com os alunos, enquanto estes conduzem suas próprias investigações e desenvolvem soluções (FRANÇA et.al., 2016).

No contexto do ensino-aprendizagem visando o desenvolvimento de soluções baseadas em software, geralmente são utilizados métodos, tanto prescritivos como ágeis, que focam na entrega de funcionalidades para atender requisitos declarados pelos usuários. Entretanto, as técnicas tradicionais que apenas perguntam às pessoas o que elas querem indicam melhorias incrementais, mas nunca leva a ideias revolucionárias capazes de mudar paradigmas e/ou reorientar um mercado (BROWN, 2009).

Segundo Borba et. al (2016), para o desenvolvimento de soluções de software inovadoras, devem ser tratados aspectos adicionais como: alinhamento com a estratégia 
de entrega de valor da organização; avaliação de soluções concorrentes; solução criativa para o problema; e entrega rápida do software com foco na proposta de valor.

A abordagem de ensino-aprendizado proposta neste trabalho consiste na aplicação de um processo de inovação para a concepção, design e experimentação de soluções baseadas em software. Do ponto de vista técnico, esta abordagem utiliza práticas e ferramentas para tratar questões não atendidas por processos de software usuais, tais como resolução criativa de problemas, análise do mercado e modelagem de negócio. Do ponto de vista humano, esta abordagem provê conhecimentos e habilidades aos estudantes necessários para o desenvolvimento de soluções inovadoras, tais como, imersão no problema antes da construção da solução, preocupação com a experiência do usuário e não somente com o uso da solução e foco na entrega de valor.

Este artigo apresenta o relato de experiência da aplicação da abordagem proposta para tratar problemas relacionados à educação. Além da introdução, este artigo possui mais três seções. A Seção 2 descreve a fundamentação teórica e metodológica que norteou o trabalho. A Seção 3 apresenta a experimentação da abordagem proposta e os resultados obtidos. A Seção 4 relata as considerações finais.

\section{Fundamentação Teórica e Metodológica}

A abordagem de ensino-aprendizagem proposta é fundamentada no paradigma PBL (Aprendizagem Baseada em Problemas). O PBL é uma abordagem instrucional centrada no estudante, que o ajuda a desenvolver o raciocínio e a comunicação, habilidades essenciais para sua vida profissional (SAVERY e DUFFY, 2001). O PBL se desenvolve em torno de um problema e visa direcionar e motivar o aprendizado de conceitos que levarão a solução do problema. O estudante é o protagonista da construção do aprendizado, que acontece em um ambiente que o imerge em atividades sobre as quais recebe feedback constante do professor e dos colegas. Ao passo que buscam a solução, os estudantes desenvolvem habilidades de trabalho em grupo, aprendizagem autônoma e atitudes como cooperação, ética e respeito a opinião alheia.

A abordagem de ensino-aprendizagem proposta objetiva a concepção, design e prototipação de soluções inovadoras baseadas em software. A diferenciação desta abordagem é a utilização de um processo de inovação para o desenvolvimento das soluções, ao invés do uso de processos de software usuais (prescritivos ou ágeis).

A metodologia de ensino consistiu na apresentação por demanda, pelo professor, dos materiais (técnicas e ferramentas com exemplos de uso) necessários para os estudantes executarem as atividades. $\mathrm{O}$ aprendizado, pelos estudantes, consistiu de um processo construtivo coletivo e colaborativo a partir do uso contextualizado do conteúdo aprendido. A abordagem proposta foi formalizada através de uma disciplina, denominada Inovação em Projetos de Software (IPS), com carga de 60 horas, executada em cursos de graduação e pós-graduação em computação. A efetividade da abordagem proposta foi avaliada pelos próprios estudantes matriculados na disciplina IPS.

O planejamento das atividades da disciplina IPS baseou-se nas fases do processo de Design (EDUCADIGITAL, 2010): Descoberta ou Imersão, para aprofundar o conhecimento no problema e entender as necessidades das pessoas; Interpretação, para analisar e sintetizar os resultados da imersão, encontrar significados e oportunidade de inovação; Ideação, para fomentar a criatividade visando geração e refinamento de ideias 
VI Congresso Brasileiro de Informática na Educação (CBIE 2017)

Anais do XXIII Workshop de Informática na Escola (WIE 2017)

de soluções; Experimentação, para construção do protótipo da solução visando a validação da ideia mais promissora a partir do feedback dos interessados; e Evolução, para planejar o aprimoramento do protótipo construído em direção ao produto final.

No contexto da disciplina IPS, os passos das fases do processo de Design foram executados com o auxílio de ferramentas fornecidas pelas seguintes abordagens de inovação complementares: Design Thinking (BROWN, 2010), a qual se preocupa com a resolução criativa de problemas; Blue Ocean Strategy (KIM e MAUBORGNE, 2005), a qual trata da avaliação de mercado através da análise das soluções concorrentes; Business Model Generation (OSTERWALDER e PIGNEUR, 2011), a qual fornece meios para a modelagem de um negócio economicamente sustentável; e Lean Startup (RIES, 2012) que estimula o pensamento empreendedor para entrega rápida de valor aos clientes. A Tabela 1 apresenta no que consiste e como foram executados os passos de cada fase do processo de Design no contexto da disciplina IPS.

Tabela 1. Detalhamento do Processo de Design

\begin{tabular}{|c|c|c|c|}
\hline Fase & Passo & O quê & Como \\
\hline \multirow[t]{3}{*}{ Descoberta } & $\begin{array}{l}\text { Entenda o } \\
\text { desafio }\end{array}$ & $\begin{array}{l}\text { Compartilhamento do } \\
\text { entendimento da equipe sobre o } \\
\text { problema }\end{array}$ & $\begin{array}{l}\text { Sessão de brainstorming } \\
\text { pela equipe do projeto }\end{array}$ \\
\hline & $\begin{array}{l}\text { Prepare a } \\
\text { pesquisa }\end{array}$ & $\begin{array}{l}\text { Elaboração de questões e seleção } \\
\text { de participantes da pesquisa }\end{array}$ & $\begin{array}{l}\text { Aplicação de um formulário } \\
\text { eletrônico da pesquisa e } \\
\text { elaboração de um Mapa } \\
\text { Mental }\end{array}$ \\
\hline & $\begin{array}{l}\text { Reúna } \\
\text { inspirações }\end{array}$ & $\begin{array}{l}\text { Aprendizado com usuários e } \\
\text { especialistas, e Avaliação do } \\
\text { mercado }\end{array}$ & $\begin{array}{l}\text { Entrevistas com } \\
\text { interessados e elaboração da } \\
\text { Matriz de Avaliação de } \\
\text { Valor }\end{array}$ \\
\hline \multirow[t]{3}{*}{ Interpretação } & Conte histórias & $\begin{array}{l}\text { Consolidação e documentação do } \\
\text { aprendizado }\end{array}$ & $\begin{array}{l}\text { Elaboração do Mapa da } \\
\text { Empatia }\end{array}$ \\
\hline & $\begin{array}{l}\text { Procure por } \\
\text { significados }\end{array}$ & Definição de insights & $\begin{array}{l}\text { Elaboração de Cartões de } \\
\text { Insights e Diagrama de } \\
\text { Afinidade }\end{array}$ \\
\hline & $\begin{array}{l}\text { Estruture } \\
\text { oportunidades }\end{array}$ & $\begin{array}{l}\text { Descrição das atividades do } \\
\text { contexto e ao redor }\end{array}$ & $\begin{array}{l}\text { Elaboração da Jornada do } \\
\text { Usuário }\end{array}$ \\
\hline \multirow[t]{2}{*}{ Ideação } & Gere ideias & $\begin{array}{l}\text { Elaboração coletiva e colaborativa } \\
\text { de ideias de solução }\end{array}$ & $\begin{array}{l}\text { Elaboração de Cardápio de } \\
\text { Ideias e execução de } \\
\text { Workshop de Cocriação }\end{array}$ \\
\hline & Refine ideias & $\begin{array}{l}\text { Seleção das ideias mais } \\
\text { promissoras }\end{array}$ & $\begin{array}{l}\text { Elaboração de Matriz de } \\
\text { Posicionamento e Curva de } \\
\text { Valor da Solução }\end{array}$ \\
\hline Experimentação & $\begin{array}{l}\text { Faça } \\
\text { protótipos }\end{array}$ & $\begin{array}{l}\text { Materialização da ideia de } \\
\text { solução }\end{array}$ & $\begin{array}{l}\text { Construção de Storytelling e } \\
\text { MVP (Produto Mínimo } \\
\text { Viável) }\end{array}$ \\
\hline
\end{tabular}


VI Congresso Brasileiro de Informática na Educação (CBIE 2017)

Anais do XXIII Workshop de Informática na Escola (WIE 2017)

\begin{tabular}{|l|l|l|l|}
\hline & $\begin{array}{l}\text { Obtenha } \\
\text { feedback }\end{array}$ & $\begin{array}{l}\text { Avaliação da solução pelos } \\
\text { interessados }\end{array}$ & $\begin{array}{l}\text { Interação com o MVP e } \\
\text { aplicação de entrevistas e } \\
\text { questionários }\end{array}$ \\
\hline Evolução & $\begin{array}{l}\text { Acompanhe o } \\
\text { aprendizado }\end{array}$ & Melhorias no MVP & $\begin{array}{l}\text { Ajustes no MVP a partir do } \\
\text { feedback dos interessados }\end{array}$ \\
\cline { 2 - 4 } & Avance & $\begin{array}{l}\text { Planejamento para transformação } \\
\text { do protótipo em produto final }\end{array}$ & $\begin{array}{l}\text { Modelagem de um negócio } \\
\text { sustentável }\end{array}$ \\
\hline
\end{tabular}

Em relação a trabalhos correlatos, há alguns relatos da aplicação Design Thinking no processo de ensino-aprendizagem. Clemente et al. (2016) apresentam a aplicação de uma variação do Design Thinking, denominado modelo Design Thinking Evolution E6 ${ }^{2}$, no desenvolvimento de uma intervenção didática no ensino superior promotora do pensamento criativo e crítico. Entre as contribuições destacadas pelos autores está a promoção das competências de pensamento e do autoconhecimento dos estudantes acerca das capacidades criativas deles. Scheer et al. (2012) relatam que há melhoria na experiência em sala de aula, tanto para professores como para estudantes, quando se utiliza Design Thinking no contexto do processo construtivista de ensino-aprendizagem. Segundo os autores, o uso do Design Thinking fomenta nos estudantes habilidades e competências exigidas para o bom desempenho das atividades do século 21 , tais como, pensamento crítico e resolução de problemas, colaboração em rede, agilidade e adaptabilidade, iniciativa, comunicação eficiente, curiosidade e imaginação.

\section{Experimentação e Resultados Obtidos}

A experimentação da abordagem de ensino-aprendizagem proposta ocorreu no contexto da disciplina IPS para turmas de graduação e pós-graduação de cursos de computação. A metodologia da disciplina IPS consistiu na execução do Processo de Design (Tabela 1) por grupos de trabalho formados pelos estudantes matriculados na disciplina. Nesta seção, inicialmente é apresentado como o processo de Design foi executado e, então, é apresentado o feedback dos estudantes sobre a abordagem adotada.

\subsection{Execução do Processo de Design}

O processo de Design consistiu na execução das seguintes fases: Descoberta, para imersão no problema e definição do desafio; Interpretação, para aprofundamento do entendimento das informações e formulação de Insights; Ideação, usar a criatividade para geração e refinamento de Ideias de Solução para o desafio; Experimentação, para materializar a ideia de solução mais promissora através do desenvolvimento de um Protótipo da Solução; e Evolução, para planejamento dos fatores-chave para tornar a solução desenvolvida parte de um Modelo de Negócios sustentável. Para execução do processo de Design consideramos um período letivo como restrição de tempo.

A fase de Descoberta iniciou com uma sessão de brainstorming na qual os membros dos grupos de trabalho compartilharam informações e experiências sobre problemas na área de educação. O objetivo foi possibilitar um entendimento comum da equipe sobre o tema em questão. Em seguida, foram projetadas questões para a pesquisa junto ao público-alvo. A pesquisa foi realizada através de realização de entrevistas e da aplicação de questionários eletrônicos cujos principais achados foram consolidados em 
VI Congresso Brasileiro de Informática na Educação (CBIE 2017)

Anais do XXIII Workshop de Informática na Escola (WIE 2017)

um Mapa Mental. O Mapa Mental é uma ferramenta de gestão do conhecimento que permite a visualização gráfica da estruturação e do relacionamento das informações.

Ao final fase de Descoberta foi elaborada uma Matriz de Avaliação de Valor (KIM e MAUBORGNE, 2005) para identificar os principais fatores-chave que são atendidos pelas soluções existentes, relacionadas ao desafio. A partir dos resultados obtidos, foi possível definir o desafio para nortear o projeto da solução. A Tabela 2 apresenta, para cada grupo de trabalho, a quantidade de membros, o nível dos cursos dos estudantes e o desafio elaborado.

Tabela 2. Formulação do Desafio

\begin{tabular}{|l|l|l|l|}
\hline Identificação & Membros & Nível & Desafio \\
\hline Grupo 1 & 4 & Graduação & $\begin{array}{l}\text { Como podemos melhorar a interação entre estudantes e } \\
\text { professores no âmbito acadêmico? }\end{array}$ \\
\hline Grupo 2 & 4 & Graduação & $\begin{array}{l}\text { Como podemos prover um meio inovador para unir } \\
\text { demanda de ensino e oferta direcionada de jogos } \\
\text { educacionais? }\end{array}$ \\
\hline Grupo 3 & 3 & $\begin{array}{l}\text { Pós- } \\
\text { graduação }\end{array}$ & $\begin{array}{l}\text { Como podemos encontrar meios de estimular estudantes e } \\
\text { professores de diferentes cursos a trabalharem de maneira } \\
\text { colaborativa no desenvolvimento de um produto ou } \\
\text { serviço, propiciando a troca de conhecimento e a } \\
\text { experiência prática voltada para o mercado de trabalho? }\end{array}$ \\
\hline
\end{tabular}

A fase de Interpretação iniciou com elaboração de um Mapa da Empatia para possibilitar um melhor entendimento sobre as principais dores (queixas) e necessidades (desejos) de cada perfil de interessado (ex: estudante e professor). Em seguida, cada grupo de trabalho debateu e definiu um conjunto de insights relacionados ao desafio. Insight é uma reflexão embasada em dados reais obtidos por meio de Pesquisas Exploratórias (VIANNA et al., 2012). Cada insight foi então descrito em um Cartão de Insight para relacioná-lo a um fato, a uma fonte e a um tema. Depois disso, foi elaborado um Diagrama de Afinidades que corresponde a um agrupamento dos cartões de insights a partir das similaridades e dependências deles. Ao final desta fase, foi elaborada uma Jornada do Usuário para permitir o entendimento das etapas percorridas pelos interessados no contexto estudado. A Tabela 3 apresenta os principais insights elaborados por cada grupo de trabalho.

Tabela 3. Elaboração de Insights

\begin{tabular}{|c|c|}
\hline Grupo & Insights \\
\hline Grupo 1 & $\begin{array}{l}\text { 1. Os professores necessitam de uma ferramenta que possibilite a obtenção de feedback } \\
\text { em tempo real dos estudantes sobre o entendimento do conteúdo ensinado; } \\
\text { 2. Os professores necessitam de uma ferramenta para tornar as aulas mais dinâmicas e } \\
\text { transmitir o conhecimento de modo inovador; } \\
\text { 3. Os estudantes necessitam de uma ferramenta de comunicação anônima para ser usada } \\
\text { durante a aula e extraclasse para fazer questionamentos e dirimir dúvidas; } \\
\text { 4. Os estudantes necessitam de uma ferramenta que disponibilize materiais didáticos que } \\
\text { os auxiliem em tempo real e sem desviar a atenção. }\end{array}$ \\
\hline
\end{tabular}


VI Congresso Brasileiro de Informática na Educação (CBIE 2017)

Anais do XXIII Workshop de Informática na Escola (WIE 2017)

\begin{tabular}{|c|c|}
\hline Grupo 2 & $\begin{array}{l}\text { 1. Os professores precisam de soluções educacionais inovadoras viáveis para usar em } \\
\text { sala de aula; } \\
\text { 2. O material tradicional usado em sala de aula pode ser adaptado com algum recurso } \\
\text { tecnológico; } \\
\text { 3. Os estudantes precisam ser estimulados a buscar aprendizados por seus próprios } \\
\text { meios; } \\
\text { 4. Os jogos são ferramentas eficazes para facilitar o aprendizado, mas não são } \\
\text { facilmente encontrados. }\end{array}$ \\
\hline Grupo 3 & $\begin{array}{l}\text { 1. A universidade precisa estabelecer processos que estabeleçam como os departamentos } \\
\text { acadêmicos podem trabalhar de maneira colaborativa; } \\
\text { 2. Os estudantes têm interesse em participar de projetos interdepartamentais, mesmo } \\
\text { sem remuneração (bolsa), com intuito de adquirir experiência; } \\
\text { 3. Os estudantes desejam construir produtos/serviços que possam beneficiá-los, bem } \\
\text { como a própria universidade; } \\
\text { 4. Os professores precisam ser incentivados a propor e trabalhar em projetos } \\
\text { interdepartamentais em suas disciplinas. }\end{array}$ \\
\hline
\end{tabular}

A fase de Ideação iniciou com uma sessão de brainstorming visando à geração de ideias para satisfazer aos insights produzidos. As ideias foram organizadas em um Cardápio de Ideias e discutidas com os interessados através de um Workshop de Cocriação. Ao final da atividade, o Cardápio de Ideias foi atualizado com novas ideias que surgiram e com aquelas que foram complementadas. Em seguida as ideias produzidas foram avaliadas por meio de uma Matriz de Posicionamento, a qual serve para identificar qual das ideias atende mais critérios norteadores. Os critérios norteadores foram definidos em termos das prioridades e desejos dos interessados, e pelos fatoreschave que orienta a área relacionada ao desafio. Ao final desta fase, foi elaborada a Curva de Valor da solução idealizada, de modo a diferenciá-la das soluções existentes (analisadas na fase de Descoberta). A Curva de Valor, descrita através de uma Matriz de Avaliação de Valor, permite uma comparação do nível de atendimento aos fatores-chave pela solução idealizada em relação às soluções "concorrentes" analisadas. A Tabela 4 apresenta a Ideia de Solução mais promissora (atende mais critérios norteadores) produzida por cada grupo de trabalho.

Tabela 4. Ideia de Solução mais promissora

\begin{tabular}{|l|l|}
\hline Grupo & Ideia de Solução \\
\hline Grupo 1 & $\begin{array}{l}\text { Sistema de Feedback da Aula: os professores apresentam questões durante a aula que } \\
\text { podem ser respondidas pelos estudantes de modo a permitir avaliar o nível de aprendizado } \\
\text { em tempo real; os estudantes introvertidos poderão postar dúvidas a serem respondidas } \\
\text { pelo professor; alinhado com o conceito de aprendizado personalizado. }\end{array}$ \\
\hline Grupo 2 & $\begin{array}{l}\text { Plataforma de Streaming de Jogos: os usuários teriam acesso aos jogos educacionais apenas } \\
\text { através da plataforma; canal entre produtores de jogos educacionais e professores que } \\
\text { precisam dinamizar e melhorar o ensino; oferta de jogos educacionais mais recomendados } \\
\text { e apropriados a uma determinada demanda de ensino/aprendizado. }\end{array}$ \\
\hline Grupo 3 & $\begin{array}{l}\text { Sistema de Registro e Compartilhamento de Propostas de Projetos: repositório de propostas } \\
\text { de projetos que podem ser desenvolvidas por iniciativas interdepartamentais; } \\
\text { recomendação personalizada de projetos para usuários de acordo com as habilidades e } \\
\text { conhecimentos exigidos; formação de times interdisciplinares para o projeto. }\end{array}$ \\
\hline
\end{tabular}


A fase de Experimentação iniciou com a elaboração de uma Storytelling para ilustrar cenários de uso para a solução idealizada. No contexto da Experimentação, a Storytelling apresenta uma narrativa sobre o problema e como a solução proposta contribui para resolver este problema através da demonstração da entrega de valor durante uma jornada do usuário. No canal YouTube estão disponíveis para visualização as Storytelling produzidas pelo Grupo 1 e Grupo 3, respectivamente, acessíveis através dos endereços eletrônicos: https://www.youtube.com/watch?v=HjcreecVRos e https://www.youtube.com/watch?v=b8MVhsmCXIs.

A fase de Experimentação prosseguiu com a elaboração de um protótipo da solução proposta, mais especificamente o MVP (Produto Mínimo Viável) da solução. O MVP é uma versão enxuta que apresenta apenas as principais funcionalidades para demonstrar a entrega de valor pelo produto. O MVP permite a obtenção rápida de feedback dos usuários sobre o produto. Nos endereços eletrônicos a seguir estão disponíveis para interação os MVP produzidos pelo Grupo 2 e Grupo 3, respectivamente: $\quad$ https://projects.invisionapp.com/share/M27PHWPPV $\mathrm{e}$ https://creator.ionic.io/share/e97c1ec799bb.

Para finalizar a fase de Experimentação, os artefatos produzidos (Storytelling e MVP) foram apresentados e disponibilizados para o público-alvo. O objetivo foi obter feedback sobre a entrega de valor da solução desenvolvida. Os grupos de trabalho aplicaram questionários eletrônicos para obter informações da percepção dos participantes sobre o problema e se a solução desenvolvida contribui com a resolução do problema. Os grupos de trabalho tiveram então subsídios para tomar a decisão de manter ou mudar a estratégia adotada. Em geral, os três grupos obtiveram resultados bastante satisfatórios a partir da avaliação pelos usuários e algumas sugestões foram consideradas para serem incorporadas nas respectivas soluções.

O processo de Design encerrou com a execução da fase de Evolução. Inicialmente, uma nova versão do MVP foi desenvolvida a partir do feedback dos usuários. Posteriormente, cada grupo eleborou um Modelo de Negócio através da ferramenta Business Model Canvas (OSTERWALDER e PIGNEUR, 2011). O Modelo de Negócio descreve: qual o valor a ser entregue ao público-alvo; qual canal será usado para permitir a entrega do valor; como se dará o relacionamento com o cliente, quais as fontes de receita; quais as principais atividades e recursos-chave necessários para o negócio operar; quais os principais custos envolvidos; e qual a rede de fornecedores e parceiros relacionados ao negócio.

\subsection{Avaliação da Disciplina IPS}

Como última atividade da disciplina IPS, foi solicitado pelo professor que os estudantes respondessem voluntariamente, e sem precisar se identificar, um questionário on-line (desenvolvido através da ferramenta Google Form). Dos onze estudantes matriculados na disciplina IPS, dez responderam o questionário.

Inicialmente foi avaliada a abordagem de ensino/aprendizagem da disciplina IPS, baseada em PBL, com baixa carga horária expositiva e com foco na experimentação prática. Considerando uma escala de 1 (Péssima) a 5 (Ótima), os estudantes avaliaram positivamente a abordagem de ensino/aprendizagem conforme apresentado na Figura 1. 


\section{Abordagem de Ensino/Aprendizagem}

\section{0 respostas}

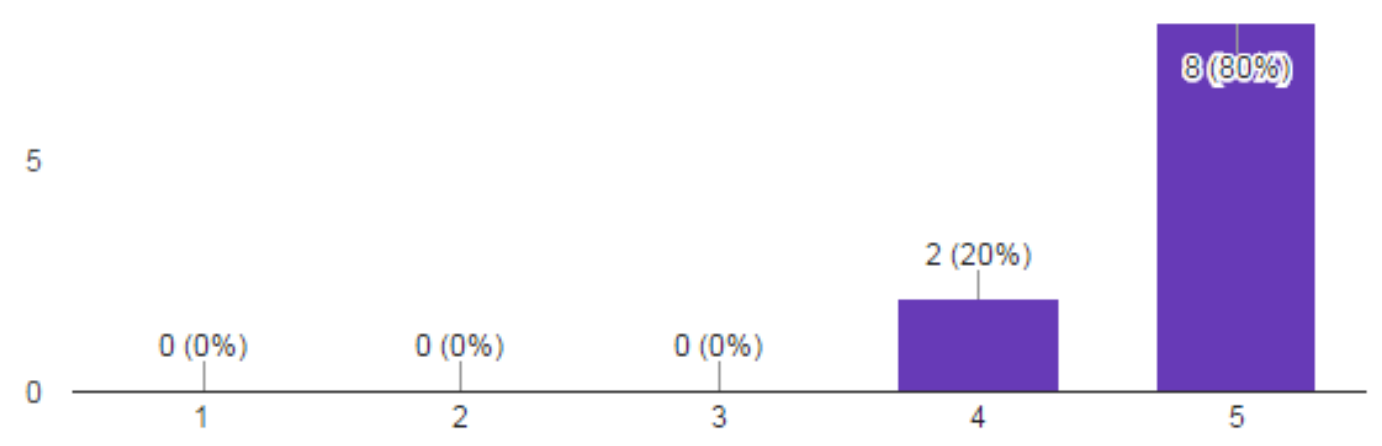

Figura 1. Avaliação da abordagem de ensino/aprendizagem pelos estudantes

Posteriormente, os estudantes avaliaram os seguintes habilidades/conhecimentos fomentados na disciplina IPS: Resolução criativa de problemas; Design inovador de solução baseada em software; Aplicação de técnicas/ferramentas centradas em inovação; Pensamento empreendedor; e Trabalho colaborativo. Para cada habilidade/ conhecimento, o estudante classificava como Aprendido/Adquirido; Melhorado; ou Não Aprendido/Não Adquirido. A Figura 2 apresenta os resultados, também positivos, da avaliação pelos estudantes das habilidades/conhecimentos fomentados na disciplina IPS.

\section{Habilidades ou conhecimentos fomentados na disciplina}

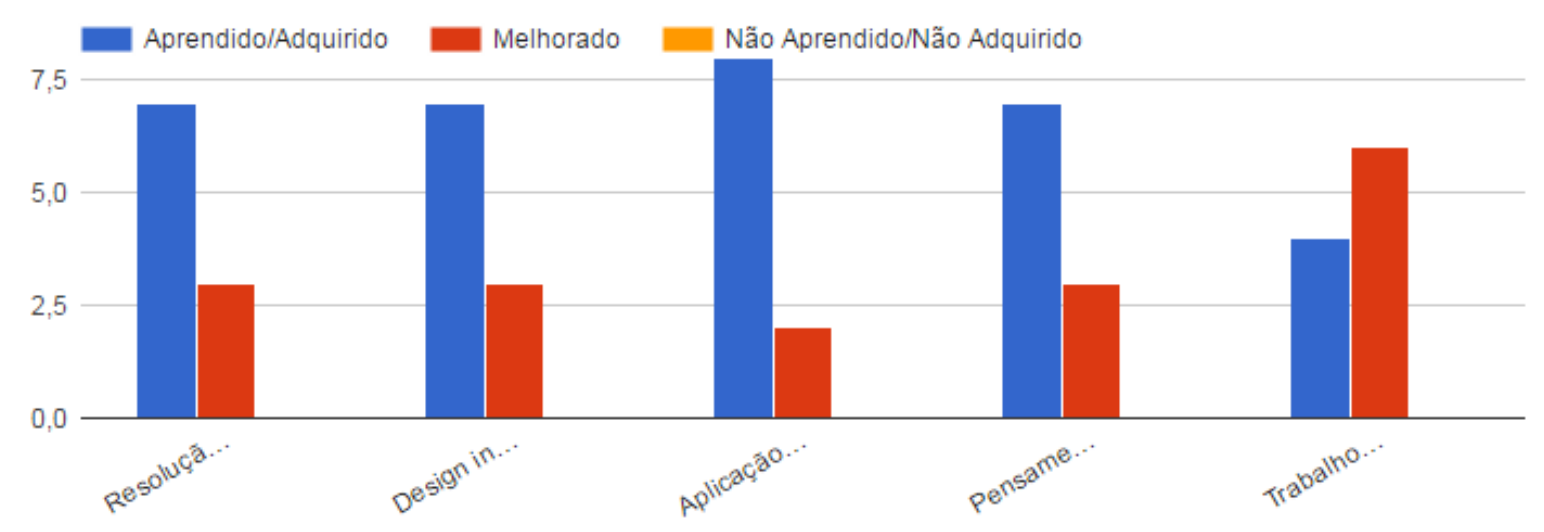

Figura 2. Avaliação de habilidades/conhecimentos pelos estudantes

Finalmente, os estudantes responderam onde pretendiam aplicar os conhecimentos e habilidades fomentados na disciplina IPS. Foram apresentadas as seguintes opções com possibilidade de múltipla escolha: Projetos de desenvolvimento de software; Pesquisas acadêmicas; Atividades acadêmicas de outras disciplinas; Resolução de problemas do cotidiano; e Outros. A Figura 3 apresenta o resultado desta avaliação pelos estudantes. 


\section{Onde pretende aplicar os conhecimento/habilidades fomentados na disciplina}

10 respostas

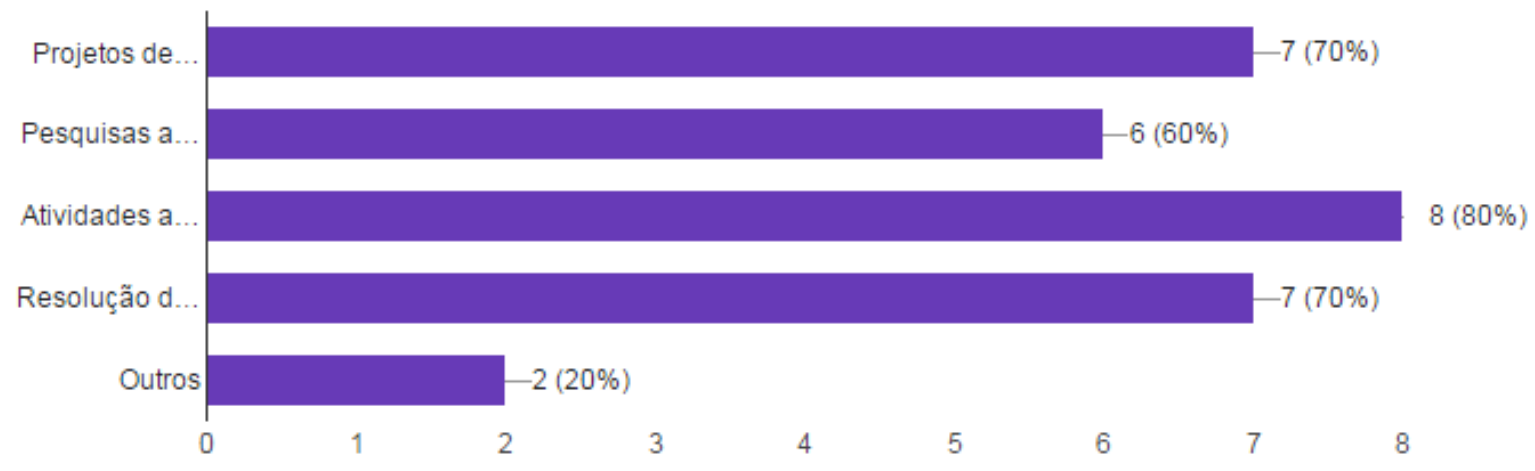

Figura 3. Avaliação pelos estudantes da aplicabilidade dos conhecimentos/habilidades fomentados na disciplinaIPS

O resultado das avaliações da disciplina IPS realizadas pelos estudantes reitera que uma abordagem de ensino-aprendizagem voltada para a resolução de um problema de maneira colaborativa é bem aceita pelos estudantes. Além disso, outra constatação, é que experiência prática de aprender através do uso fomenta habilidades/conhecimentos que são exigidos nos trabalhos atuais, tais como, a criatividade para resolução de problemas, o trabalho em equipe e o pensamento empreendedor voltado para o design de soluções economicamente sustentáveis. Por último, os conhecimentos/habilidades adquiridos/melhorados pelos estudantes tem aplicabilidade em outros contextos, tanto acadêmico, como profissional e até mesmo pessoal (dia-a-dia).

\section{Considerações Finais}

O ensino tradicional de engenharia de software inicia com a especificação dos requisitos junto aos usuários, e o restante do processo consiste em projetar, implementar e testar funcionalidades que atendam a estes requisitos. Este tipo de estratégia leva a produtos de software para dar suporte a processos de negócios estabelecidos, mas dificilmente reorientam um mercado ou atendem uma demanda latente (BORBA et. al, 2016).

Em busca da inovação, faz-se necessário: entendimento profundo do problema para identificar desafios relevantes; geração de ideias criativas para solução do problema com a participação ativa dos interessados; materialização da ideia mais promissora por meio de protótipos visando a obtenção de feedback sobre a solução projetada; e tornar a solução parte de um modelo de negócios sustentável.

Diante deste cenário, este artigo apresentou o relato de experiência da aplicação de uma abordagem de ensino-aprendizagem, baseada no PBL e orientada pelo processo de Design, para o desenvolvimento de soluções inovadoras baseadas em software. A abordagem de ensino-aprendizagem proposta foi validada no contexto da disciplina de Inovação em Projetos de Software (IPS), executada em nível de graduação e pósgraduação, na qual os estudantes trabalharam em equipe visando projetar soluções para resolver problemas relacionados à educação. 
VI Congresso Brasileiro de Informática na Educação (CBIE 2017)

Anais do XXIII Workshop de Informática na Escola (WIE 2017)

Além dos conhecimentos técnicos, a abordagem proposta objetivou prover habilidades aos estudantes necessárias para a busca pela inovação, tais como, criatividade na resolução de problemas, pensamento empreendedor e trabalho colaborativo. Neste sentido, a abordagem se mostrou satisfatória com base em avaliações realizadas pelos próprios estudantes ao final da experiência. Este trabalho limitou-se em apresentar a fundamentação teórico-metodológica e a experimentação da abordagem de ensinoaprendizagem proposta. A execução detalhada do processo de Design, em termos dos artefatos produzidos, pode ser consultada em Silva et. al (2016), que relata a experiência de um dos grupos de estudantes citados neste trabalho.

\section{Referências}

Borba, A.; Batista, G.; Souza, R. (2016). InnoStartup: a Toolbox for Innovation in Software Development Process. IEEE Latin America Transactions, Vol. 14, No. 8.

Brown, T. (2010). Design Thinking: uma metodologia poderosa para decretar o fim das velhas ideias. Rio de Janeiro, RJ: Elsevier.

Clemente, V.; Tschimmel, K.; Vieira, R. (2016). Pensamento criativo e crítico no Desenvolvimento de Produto: uma intervenção didática baseada no Design Thinking. Revista Lusófona de Educação, 32(32), 75-92.

Educadigital, I. (2010). Kit de Design Thinking para Educadores. Disponível em http://www.dtparaeducadores.org.br/. Acessado em Junho de 2016.

França, C.; Falcão, T.; Marinho, M.; Cardoso, M.; Nóbrega, O.; Souza. R; Sampaio, S. (2016). EITA! Uma Proposta Inovadora para um Programa de Pós-Graduação LatuSensu em Educação, Inovação e Tecnologia Aplicada. Anais do XXII Workshop de Informática na Escola (WIE 2016).

Kim C.; Mauborgne, R. (2005). A Estratégia do Oceano Azul: como criar novos mercados e tornar a concorrência irrelevante. Rio de Janeiro, RJ: Elsevier.

OECD (2014). "Measuring Innovation in Education: A New Perspective, Educational Research and Innovation", OECD Publishing. DOI: 10.1787/9789264215696-en.

Osterwalder, A.; Pigneur, Y. (2011). Business Model Generation: Inovação em Modelos de Negócio. Rio de Janeiro, RJ: Alta Books.

Ries, E. (2012). A Startup Enxuta. São Paulo, SP: Lua de Papel.

Savery, J.; Duffy, T. (2001). Problem Based Learning: An instructional model and its constructivist framework. CRLT Technical Report No. 16-01. Indiana University.

Scheer, A.; Noweski, C.; Meinel, C. (2012). Transforming Constructivist Learning into Action: Design Thinking in education. Design and Technology Education: An International Journal, 17(3), 8-19.

Silva, A.; Correa, A.; Souza Filho, J.; Souza, R. (2016). Aplicação do Design Thinking em um Problema Educacional: Um Relato de Experiência. Anais do XXII Workshop de Informática na Escola (WIE 2016).

Vianna, M.; Vianna, Y.; Adler, I.; Lucena, B.; Russo, B. (2012). Design Thinking: inovação em negócios. Rio de Janeiro, RJ: MJV Press. 